\title{
Steering a Leader-Follower Team via Linear Consensus
}

\author{
Fabio Pasqualetti, Simone Martini, and Antonio Bicchi \\ Università di Pisa, Interdipartimental Research Center "E. Piaggio", Italy, \\ Facoltà di Ingegneria, Università di Pisa, via Diotisalvi, 2 \\ 56126 Pisa, Italy \\ fabiopass@gmail.com, s.martini@ingegneria.pisa.it, bicchi@ing.unipi.it, \\ wWw.piaggio.ccii.unipi.it
}

\begin{abstract}
The paper considers the problem of driving a formation of autonomous mobile agents. The group of mobile devices is represented by a leader-follower network, where the followers update their position using a simple local consensus procedure, while the leaders, whose positions represent the control inputs of the network, are free to move. We characterize the transient behavior of the network, and we solve the containment problem without relying on auxiliary sensors.
\end{abstract}

\section{Introduction}

In an increasing number of applications in robotics, surveying, industrial automation, etc., the use of large teams of networked mobile agent systems is being proposed to achieve effectiveness and robustness to failures of single agents. In these schemes, teams (also referred to as formations, or flocks, etc.) coordinate their motion based on local interactions between neighbors, without referring to a centralized authority. An important class of decentralized motion control strategies is that of consensus algorithms (see e.g. $[1,2]$ ), for which a rather well established theory is available that enables a thorough analysis of convergence properties. In this paper we consider the steering problem, i.e. that of leading a team of autonomous agents using only local interactions and communications, in the accomplishment of a task where some degree of global information (e.g. on the environment) is necessary. To this purpose, we refer to a leader-follower structure, whereby a (small) subset of agents is assumed to have access to global information, and to lead through local consensus interactions the remaining agents. We adopt the team structure proposed in [3], which is particularly suitable when the goal is to steer a group of agents while maintaining certain geometric properties. For instance, if the agents transport some dangerous materials, it is important to keep them inside a proper area, not to contaminate the outer region. In [3], authors investigate properties such as controllability, containment and optimal control of leader - follower consensus structure for steering a group of mobile agents. Containment techniques rely on 
the use of sensors to detect the movements of the driven agents, and to trigger hybrid control actions by the team leaders. Several interesting problems remain open in leader-follower networks, including e.g. a characterization of the equilibrium manifold for a team with a given connectivity graph, and the minimal number of leaders necessary to achieve a specified task. The contribution of this short paper consists of solving the containment problem by characterizing the convergence speed of the consensus law implemented by the followers, and hence allows to use a containment technique without recurring to additional sensors to test the presence of the agents in the desired region.

\section{Leader - Follower Consensus Networks}

Consider a set $V=\{1, \ldots, n\}$ of mobile agents, communicating as described by a directed graph $G_{r}$. The digraph is defined by the triple $(V, \mathcal{E}, H)$, being $\mathcal{E}$ a set of edges, and $H$ an $n \times n$ weighted adjacency matrix whose entries satisfy $h_{k j}>0$ if the pair $(j, k) \in \mathcal{E}$. The agents $V$ are partitioned into an $m$ dimensional leader set $L$, and a follower set $F$, so that $L \cap F=\emptyset$, and $L \cup F=V$. Leaders and followers differ in their motion law. Indeed, the former arbitrarily update their positions while the latter use a linear consensus law described by the $n-m \times n$ stochastic matrix $\left[\begin{array}{ll}A & B\end{array}\right]$. The equation describing the motion along the $X$ axis of the followers is $x_{F}(t+1)=A x_{F}(t)+B x_{L}(t)$, where $x_{F}$ and $x_{L}$ collect respectively the followers and the leaders positions. Since analogous considerations can be made for the motion along the $Y$ axis, in the rest of the paper we only focus on the $X$ dimension. Note that, if $G_{r}$ is connected, the matrix $A$ is stable, so that, when $x_{L}$ is constant, the system reaches an equilibrium configuration described by $\bar{x}_{F}=(I-A)^{-1} B x_{L}$.

\section{The Containment Problem}

We define the containment problem as the problem of driving a collection of autonomous mobile agents (followers) to a given target location, while guaranteeing that their motion remains confined in the smallest rectangle $R_{L}$ containing the positions of the leaders. It has already been shown ([3]) that, in the equilibrium, the followers are contained in the convex hull defined by the positions of the leaders, and hence in $R_{L}$. However, if the motion of the leaders is not properly controlled, some followers could exit the region $R_{L}$ during the process. For simplicity, but without loss of generality, we assume that the leaders coordinate their motion in a way that the sequence of vertexes defining the convex hull remains the same during the maneuver.

Let $x=\left(x_{F}, x_{L}\right)^{T}$, and $\delta(t)=x(t+1)-x(t)$. The vector $\delta(0)$ contains the initial movements of the agents, which are 0 for the followers, and $\delta_{0}$ for the leaders, where $\delta_{0}$ is the maximum leaders velocity that ensures containment. 
The evolution of $\delta$ is $\delta(t+1)=\left[\begin{array}{cc}A & B \\ 0 & I\end{array}\right] \delta(t)$. When $t$ grows, we have

$$
\lim _{t \rightarrow \infty} \delta(t)=\lim _{t \rightarrow \infty}\left[\begin{array}{cc}
A & B \\
\mathbf{0} & I
\end{array}\right]^{t} \delta(0)=\left[\begin{array}{cc}
0 & (I-A)^{-1} B \\
0 & I
\end{array}\right] \delta(0)=\delta_{0} \mathbf{1},
$$

where the last passage holds because $\left[\begin{array}{ll}A & B\end{array}\right]$ is stochastic. It follows that, when the leaders move at constant velocity, the group of agents behaves asymptotically as a flock, where all the agents move at the same speed. The convergence speed of the variable $\delta$ is dictated by the matrix $A$. Define $\rho$ as the spectral radius of $A$, and consider the consensus error of the follower $i$ as $\varepsilon_{i}(t)=\delta_{0}-\delta_{i}(t)$. Since $\varepsilon_{i}$ converges exponentially fast, it can be upper bounded by $\rho^{t} \delta_{0}$. Consequently, it must be $\sum_{t=0}^{\infty} \delta_{0}-\delta_{i}(t)<d$, where $d$ is the minimum among the distances between the followers and the leaders. We obtain $\sum_{t=0}^{\infty} \rho^{t} \delta_{0} \leq d$. Because of the stability of $A$, we have $\sum_{t=0}^{\infty} \rho^{t}=(1-\rho)^{-1}$, and finally

$$
\delta_{0} \leq \frac{d}{(1-\rho)^{-1}}
$$

Condition (1) characterize the maximum constant velocity of the leaders that guarantees the containment property. We might also be interested in ensuring that the connectivity in the network is maintained during the motion. In that case, let $r$ be the communication range, then

$$
\delta_{0} \leq \frac{\min \left(d, d_{r}\right)}{(1-\rho)^{-1}}, \quad d_{r}=r-d .
$$

For completeness, we describe an upper bound for $\rho$.

Theorem 1 (Leader - Follower Convergence Rate). Let $G$ be a Leader Follower consensus network with matrices $A$ and $B$, then $\rho<\left(1-w_{\text {min }}^{l}\right)^{1 / l}$, where $\rho$ is the spectral radius of $A$, and $w_{\text {min }}, l$ are respectively the minimum weight of the edges in the network and the diameter of the communication graph.

Proof. We use the same procedure as in [4]. Note that it takes at most $l$ steps to reach a leader state from a follower state with probability not less than $w_{\min }^{l}$. Since $\rho \leq\left\|A^{k}\right\|^{1 / k}, \forall k \in \mathbb{Z}_{>0}$, then $\rho \leq\left\|A^{k}\right\|_{\infty}^{1 / k}<\left(1-w_{\min }^{l}\right)^{1 / l}$.

\section{A Simulation Study}

As an example consider a swarm of helicopters consisting of 20 agents. For some strategic reasons, the optimal disposition of the followers is a double-layer pentagon, as in Fig. 1(c). The leaders have to maintain such structure while moving among obstacles toward a target location. Moreover, they know the trajectory to follow, and they adjust the size of their steps in order to guarantee the containment and the connectivity properties as in (2). In Fig. 4 some steps of the steering process are reported. Starting from the initial situation of Fig. 1(b), leaders create the desired followers formation, and steer the swarm. Because of the presence of some obstacles, the formation is shaped as in Fig. 1(e), and eventually recovered (Fig. 1(f)). 


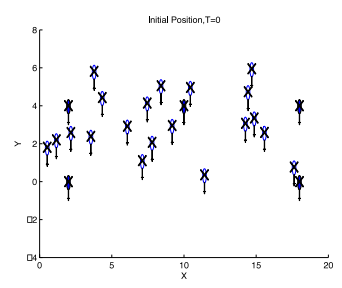

(a)

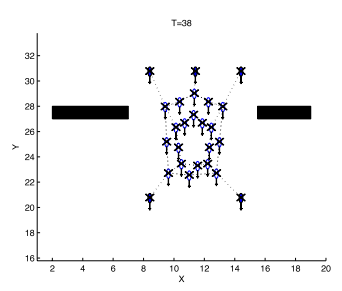

(d)

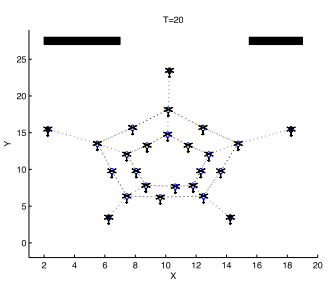

(b)

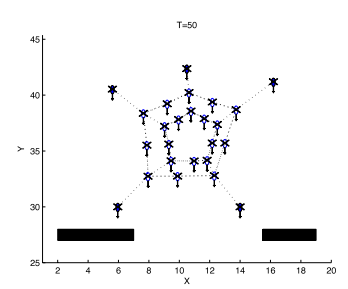

(e)

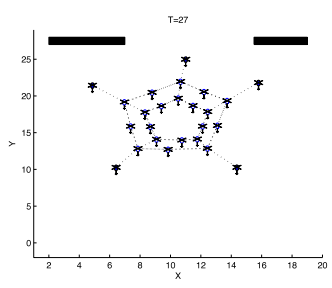

(c)

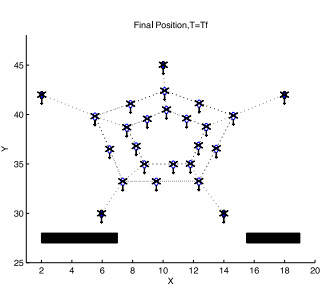

(f)

Fig. 1. Safe steering of a group of vehicles.

\section{Conclusions and Future Work}

In this short paper, we have shown that the requirement of using sensors to detect transitions of follower agents outside a guarded region in a leader-follower containment problem can be removed, provided that leaders choose their velocity according to bounds that depend on the consensus network convergence properties. Future work will address the investigation of other properties of such systems, including a characterization of the geometry of the steerable manifold for a general leader-follower network, and the study of the minimal number of leaders required to achieve given geometric properties.

\section{References}

1. J. A. Fax and R. M. Murray. Information flow and cooperative control of vehicle formations. IEEE Transactions on Automatic Control, 49(9):1465-1476, 2004.

2. R. Olfati-Saber, J. A. Fax, and R. M. Murray. Consensus and cooperation in networked multi-agent systems. IEEE Proceedings, 95(1):215-233, 2007.

3. G. Ferrari-Trecate, M. Egerstedt, A. Buffa, and M. Ji. Laplacian sheep: A hybrid, stop-go policy for leader-based containment control. Hybrid Systems: Computation and Control, Santa Barbara, CA, March 2006.

4. F. Pasqualetti, A. Bicchi, and F. Bullo. Distributed intrusion detection for secure consensus computations. IEEE Conf. on Decision and Control, 2007. 